\title{
Using a Graphical Method to Assist the Evaluation of Complicated Patterns of Evidence
}

REFERENCE: Dawid AP, Evett IW. Using a graphical method to assist the evaluation of complicated patterns of evidence. J Forensic Sci 1997;42(2):226-231.

\begin{abstract}
The forensic scientist often faces the task of interpreting patterns of evidence which involve many variables. Combining different items of evidence within a complex framework of circumstances requires logical powers of reasoning and this can be assisted by formal methods. We discuss one such method which, as has already been pointed out by Aitken and Gammerman (1), offers considerable potential for creating probabilistic expert systems to assist in evidence interpretation. In particular, we show how the method, which is based on a directed acyclic graph, enables dependencies between different aspects of the evidence to be considered. The discussion is based on an imaginary case example.
\end{abstract}

KEYWORDS: forensic science, interpretation, expert systems, probability, Bayesian, fibers, trace evidence

There is now considerable support for the Bayesian approach to assessing evidence in the context of a legal adversary system (2-5). The examples which are described in the literature tend, of necessity, to be relatively simple and practical complications are minimized so that the principles of the method are not obscured. A major factor in evaluating more complex patterns of evidence is the problem of understanding all of the dependencies which may exist between different aspects of the evidence. In this paper, we show how a graphical method, based on an approach previously applied to forensic problems by Aitken and Gammerman (1), provides a valuable aid. We base our discussion on an example and follow a manual approach but we agree with the previous authors that a computer solution is desirable. One of us has developed such solutions in the field of medical diagnosis (Spiegelhalter et al. (6)) and in a future paper we hope to demonstrate practical application of such a program.

\section{The Principles of Analysis Using a Directed Acyclic Graph}

A criminal case will often involve various items of evidence of different kinds, related to each other in more or less complex ways. For example, we might have forensic evidence concerning both bloodstains left at the crime scene and fibers found on a suspect; eyewitness evidence from a victim or a passer-by; alibi evidence; etc. The relationships between these items may depend on whether

'Professor, Department of Statistical Science Service, University College London, UK.

${ }^{2}$ Head of Interpretation Research, Forensic Science Service, Birmingham, UK.

Received 23 April 1996; and in revised form 2 Aug. 1996; accepted 7 Aug. 1996. or not the suspect is truly the offender, because the explanations of how the evidence came to be found will differ given the two different sets of circumstances. In complicated case, it can be an aid to understanding and analysis to represent these relationships in the form of a suitable graphical diagram. An early approach of this kind was Wigmore's chart method $(7,8)$. Such diagrams can make it much easier to appreciate the logical structure of a complex case.

The value of a graphical representation, however, goes far beyond its use to assist human comprehension. It can also be used to structure and organize the calculations required to find the allimportant likelihood ratio $(3,4)$ between the defense and prosecution propositions based on the totality of the evidence with all its complicated pattern of interactions. In recent years there has been much research in the statistics and artificial intelligence (AI) communities into computerized methods for probability calculations in graphically specified problems, going under the name probabilistic expert systems (PES) as described by Buckleton and Walsh (9). It is our belief that such systems, if developed further to incorporate the special features of forensic inference, could revolutionize the practice of forensic science, by making feasible a full and reasoned assessment of the overall impact of the evidence.

Graphical Representation-A PES is typically represented graphically by drawing a "node" for each variable in the problem, and arrows between certain pairs of nodes. The variables may be quantitative (e.g., a measurement), qualitative (e.g., hair color) or binary (e.g., presence/absence at the scene of crime). It is important to include not only those variables whose values are known, and thus part of the evidence, but also any others (even though unobserved) on which observed variables may reasonably be considered to depend. The arrows between variables represent the intuitive notion of "causal dependence," although this should be understood in a nondeterministic, probabilistic sense, as in the phrase "smoking causes lung cancer." Thus arrows are drawn coming into any node from the set of those other nodes which can be regarded, singly or jointly, as influencing, in a probabilistic way, its value.

\section{Example}

An unknown number of offenders entered commercial premises late at night through a hole which they cut in a metal grille. Inside, they were confronted by a security guard who was able to set off an alarm before one of the intruders punched him in the face, causing his nose to bleed.

The intruders left from the front of the building just as a police patrol car was arriving and they dispersed on foot, their getaway car having made off at the first sound of the alarm. The security guard said that there were four men but the light was too poor for 
him to describe them and he was confused because of the blow he had received. The police in the patrol car saw the offenders only from a considerable distance away. They searched the surrounding area and, about 10 min later, one of them found the suspect trying to "hot wire" a car in an alley about a quarter of a mile from the incident.

At the scene, a tuft of red fibers was found on the jagged end of one of the cut edges of the grille. Blood samples were taken from the guard and the suspect. The suspect denied having anything to do with the offense. He was wearing a jumper and jeans which were taken for examination.

A spray pattern of blood was found on the front and right sleeve of the suspect's jumper. The blood type was different from that of the suspect, but the same as that from the security guard. The tuft from the scene was found to be red acrylic. The suspect's jumper was red acrylic. The tuft was indistinguishable from the fibers of the jumper by eye, microspectrofluorimetry and thin layer chromatography (TLC). The jumper was well worn and had several holes, though none could clearly be said to be a possible origin for the tuft.

We can summarize the salient features of the evidence against the suspect as follows: G: the evidence of the security guard, W: the evidence of the police officer who arrested the suspect, and $\mathrm{R}$ : the bloodstain in the form of a spray on the suspect's jumper.

We use $X_{i}$ terms to summarize the evidence afforded by measurements on samples of known origin: $X_{1}$ : suspect's blood type, $X_{2}$ : guard's blood type, and $X_{3}$ : properties of the suspect's jumper.

And $Y_{i}$ terms similarly to summarize measurements on samples of unknown origin: $Y_{1}$ : properties of fiber tuft, and $Y_{2}$ : blood type of blood spray on jumper.

So far these are all variables which have been observed. It is helpful to make a distinction between the name for a variable and the value that it actually takes. We will denote each observation by the lower case equivalent of the variable name (i.e., $g, w, r, x_{1}$ . . . etc.) to remind us that other observations might have been made. There are other variables which are essential to the evaluation, but which have not been observed: $C$ : whether the suspect was or was not one of the offenders. We denote the values it can take as $c$, suspect was one of the offenders, and $\bar{c}$, suspect was not one of the offenders. A: the identity of the person who left the fibers on the grille. We use $a$ to denote that it was the suspect, and $\bar{a}$ that it was someone else. B: the identity of the person who punched the guard. We use $b$ to denote that it was the suspect, and $\bar{b}$ that it was someone else. $\mathrm{N}$ : the number of offenders.

If the suspect is taken to court, then it is necessary to use the above evidence to weigh against each other the two uncertain alternatives $c$ and $\bar{c}$. Formally, this means assigning the odds against $c$ conditioned on the observed evidence:

$$
\frac{P\left(c \mid g, w, r, x_{1}, x_{2}, x_{3}, y_{1}, y_{2}\right)}{P\left(\bar{c} \mid g, w, r, x_{1}, x_{2}, x_{3}, y_{1}, y_{2}\right)} \text {. }
$$

For the purposes of this paper, we are concerned with the evaluation of the scientific evidence and we can separate it from the other evidence by means of Bayes' theorem, as follows:

$\underline{P\left(c \mid g, w, r, x_{1}, x_{2}, x_{3}, y_{1}, y_{2}\right)}$

$\overline{P\left(\bar{c} \mid g, w, r, x_{1}, x_{2}, x_{3}, y_{1}, y_{2}\right)}$

$=\frac{P\left(r, x_{1}, x_{2}, x_{3}, y_{1}, y_{2} \mid c, g, w\right)}{P\left(r, x_{1}, x_{2}, x_{3}, y_{1}, y_{2} \mid \bar{c}, g, w\right)} \frac{P(c \mid g, w)}{P(\bar{c} \mid g, w)}$
We are principally concerned with the first term on the right hand side: the likelihood ratio (LR). In its present form, there is nothing that can be done to assign the two probabilities because the conditioning is far too complex. It is necessary to decompose them into simpler components but this process depends crucially on dependencies which may exist between the various aspects of the conditioning evidence. One way of understanding and displaying the dependencies in a given problem is by means of graphical representation. We do this now using a directed acyclic graph, following the approach which was first suggested in the forensic context by Aitken and Gammerman (1). This can be seen as Fig. 1 , whose explanation is as follows:

The letters denote the variables: Those that have been observed are shown in squares; those which are unobserved are shown in circles. A distinction is made between the two components of the guard's evidence: $G_{1}$ is his recollection of the number of offenders; and $G_{2}$ is his evidence that he was punched, causing his nose to bleed. If he had also provided evidence with regard to the identity of the offenders, then that could have been included as another component, $G_{3}$ perhaps. We have included $N$ as an "unobserved variable' even though the guard said there were four men, because he might been mistaken given all of the confusion surrounding the incident. However, we regard $G_{2}$ as truthful evidence of the facts it reports, and uninformative about anything else (e.g., the identity of the puncher). Although the graph could be elaborated to include nodes for these underlying facts, in addition to the guard's report of them (similarly to the introduction of node $N$ ), it is in this case equivalent and simpler just to regard $G_{2}$ as denoting those facts.

The arrows denote dependencies. Each variable is dependent only on variables which precede it in the sense indicated by the arrows. The graph appears forbidding at first sight but it can be understood by working through the various paths from the bottom up. For example, $Y_{2}$, the measurement of the blood type of the spray on the jumper is dependent on $X_{1}$, the suspect's blood type (because it might be a self stain) and the guard's blood type $X_{2}$. But information is also provided by $R$, the variable which describes the shape of the stain, because that sheds light on whether or not it might be a self stain. In turn, the shape of the stain is influenced by the way in which the guard was punched, $G_{2}$, and $B$, the identity of the person who did it. $B$ is in turn influenced by whether or not the suspect was one of the offenders, variable $C$, and also the number of offenders, $N$.

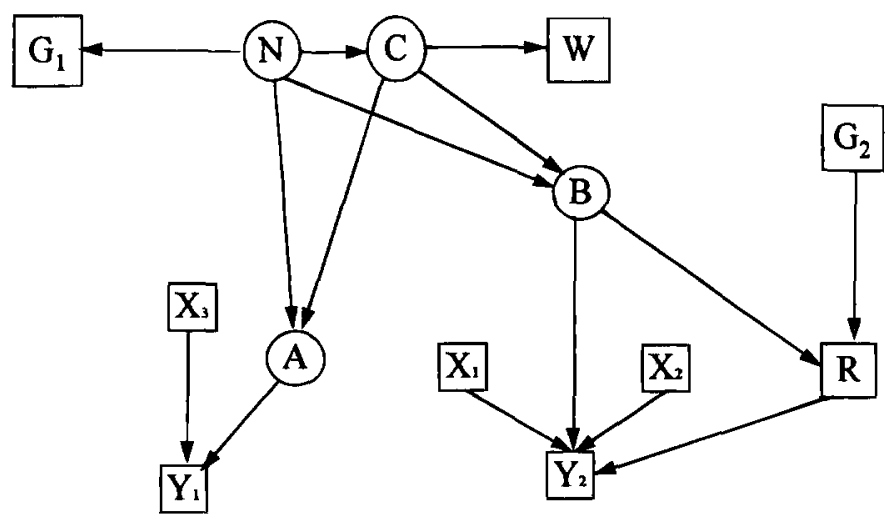

FIG. 1-Directed acyclic graph which shows the dependencies between the various aspects of the evidence. 
Although the graph exhibits many assumptions about dependencies, it contains even more implied assumptions about independencies. Thus, for example, we are assuming that the suspect's blood type $X_{1}$ is independent of the guard's type $X_{2}$, something that would not be justified if they were related, for example.

The graph also illustrates the notion of conditional independence. Thus, for example, if the identity of the person who struck the guard, $B$ is known, then $G_{1}, N, C$, and $W$ are irrelevant to any variables which are "descendants" of $B$ in the graph, for instance $Y_{2}$. This can be written in shorthand as $Y_{2} \Perp\left(G_{1}, N, C, W\right) \mid B$.

There is a straightforward way of querying the graph to determine exactly what further properties of conditional independence between various variables it embodies (10). We describe this by illustration. Suppose we wish to know whether the property $(B$, $R) \perp \perp\left(G_{1}, Y_{1}\right) \mid(A, N)$ holds. We first construct the subset of Fig. 1 containing all the nodes under consideration, together with their "ancestors" in the graph. This yields the "ancestral subgraph" in Fig. 2.

Next we note that some nodes have "unmarried parents," e.g., $A$ and $X_{3}$ in the case of $Y_{1}$. We therefore "moralize" the graph by adding additional links between all unmarried parents of the same child. This delivers the "moral ancestral graph" of Fig. 3, in which the original arrows can now be dropped.

Finally, to test for $(B, R) \perp\left(G_{1}, Y_{1}\right) \mid(A, N)$, we see whether it is possible to follow a continuous path in the moral ancestral graph, joining a node in $(B, R)$ to one in $\left(G_{1}, Y_{1}\right)$, without ever passing through $A$ or $N$. If this is not possible, we can deduce that the considered independence property does indeed follow from the assumptions embodied in Fig. 1. In fact, it is readily seen to be impossible in this case, so that we can assert $(B, R) \perp\left(G_{1}\right.$, $\left.Y_{1}\right) \mathrm{l}(A, N)$.

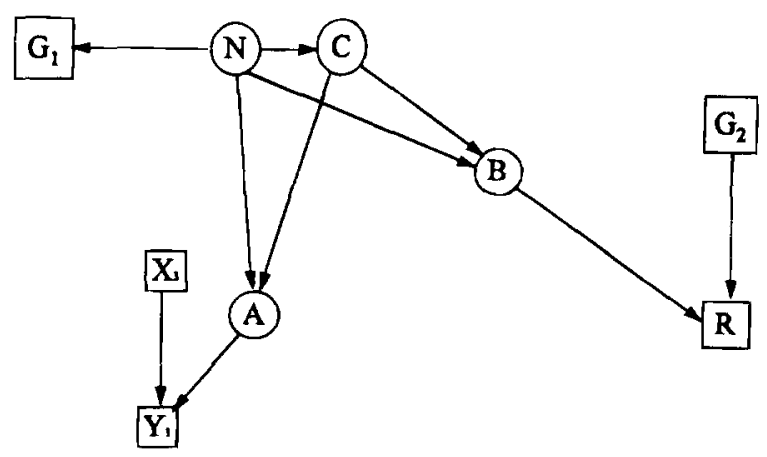

FIG. 2-Ancestral subgraph for the illustration of $(\mathrm{B}, \mathrm{R}) \perp \perp$ $\left(G_{1}, Y_{1}\right) \mid(A, N)$.

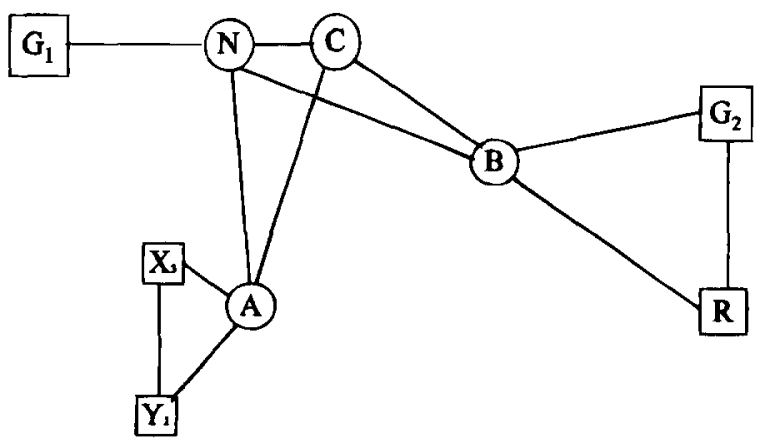

FIG. 3-Moral ancestral subgraph for the illustration of $(\mathrm{B}, \mathrm{R}) \perp$ $\left(\mathrm{G}_{1}, \mathrm{Y}_{1}\right) \mid(\mathrm{A}, \mathrm{N})$.
Had we instead tried to show $(B, R) \perp \perp\left(G_{1}, Y_{1}\right) \mid(A)$ we should have failed: the moral ancestral graph is unchanged, and in the path $B-N-G_{1}$ joins $(B, R)$ to $\left(G_{1}, Y_{1}\right)$ without passing through $A$. Hence this conditional independence property can not be asserted.

A similar, but simpler, test is available for independence: thus $\left.X_{3}\right\lrcorner \perp\left(B, G_{2}\right)$, because, in the relevant moral ancestral graph (Fig. $4)$, there is no path at all joining $X_{3}$ to a node in $\left(B, G_{2}\right)$.

\section{Simplification of the $L R$} have:

Applying Bayes' Theorem conditionally on $\left(c, g_{1}, g_{2}, w\right)$ we

$$
\begin{aligned}
& \frac{P\left(r, x_{1}, x_{2}, x_{3}, y_{1}, y_{2} \mid c, g_{1}, g_{2}, w\right)}{P\left(r, x_{1}, x_{2}, x_{3}, y_{1}, y_{2} \mid \bar{c}, g_{1}, g_{2}, w\right)} \\
& =\frac{P\left(r, y_{1}, y_{2} \mid x_{1}, x_{2}, x_{3}, c, g_{1}, g_{2}, w\right)}{P\left(r, y_{1}, y_{2} \mid x_{1}, x_{2}, x_{3}, \bar{c}, g_{1}, g_{2}, w\right)} \frac{P\left(x_{1}, x_{2}, x_{3} \mid c, g_{1}, g_{2}, w\right)}{P\left(x_{1}, x_{2}, x_{3} \mid \bar{c}, g_{1}, g_{2}, w\right)}
\end{aligned}
$$

Also, by applying the above methods the graph can be used to show that $\left(X_{1}, X_{2}, X_{3}\right)$ are independent of $\left(C, G_{1}, G_{2}, W\right)$ so the second ratio in the right hand side is one. The LR is now:

$$
\frac{P\left(r, y_{1}, y_{2} \mid x_{1}, x_{2}, x_{3}, c, g_{1}, g_{2}, w\right)}{P\left(r, y_{1}, y_{2} \mid x_{1}, x_{2}, x_{3}, \bar{c}, g_{1}, g_{2}, w\right)}
$$

However, the graph also shows that $\left(R, Y_{1}, Y_{2}\right) \perp W\left(C, X_{1}, X_{2}\right.$, $X_{3}, G_{1}, G_{2}$ ) so that $w$ can be dropped from this expression. Also, it will simplify things to assume that the guard's evidence with regard to the number of offenders (call it $n$ for the time being) is completely reliable. These two sentences lead to the following LR:

$$
\frac{P\left(r, y_{1}, y_{2} \mid x_{1}, x_{2}, x_{3}, c, n, g_{2}\right)}{P\left(r, y_{1}, y_{2} \mid x_{1}, x_{2}, x_{3}, \bar{c}, n, g_{2}\right)}
$$

Now from Fig. 1 we can establish the independence of $Y_{1}$ and $(R$, $\left.Y_{2}\right)$, given $\left(X_{1}, X_{2}, X_{3}, C, N, G_{2}\right)$. Hence the numerator of (2) factorizes as:

$$
P\left(y_{1} \mid x_{1}, x_{2}, x_{3}, c, n, g_{2}\right) P\left(r, y_{2} \mid x_{1}, x_{2}, x_{3}, c, n, g_{2}\right)
$$

Further, we can see that $\left.Y_{1}\right\lrcorner \perp\left(X_{1}, X_{2}, G_{2}\right) \mid\left(X_{3}, C, N\right)$, so that the first factor in (3) simplifies to $P\left(y_{1} \mid x_{3}, c, n\right)$. Also, $\left(R, Y_{2}\right) \perp \perp$ $\left(X_{3}\right) \mid\left(X_{1}, X_{2}, C, N, G_{2}\right)$ so the second factor simplifies to $P\left(r, y_{2} \mid x_{1}\right.$, $\left.x_{2}, c, n, g_{2}\right)$. Thus we have:

$$
\begin{gathered}
P\left(r, y_{1}, y_{2} \mid x_{1}, x_{2}, x_{3}, c, n, g_{2}\right) \\
=P\left(y_{1} \mid x_{3}, c, n\right) P\left(r, y_{2} \mid x_{1}, x_{2}, c, n, g_{2}\right)
\end{gathered}
$$

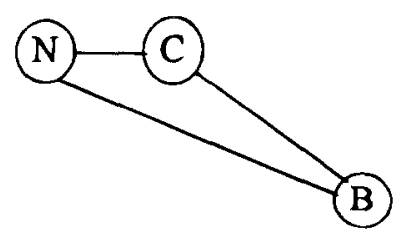

$\mathrm{G}_{2}$

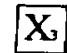

FIG. 4-Moral ancestral subgraph for the illustration of $\mathrm{X}_{3} \Perp\left(\mathrm{B}, \mathrm{G}_{2}\right)$. 
Because a similar factorization holds for the denominator of (2), we can factorize the LR as:

$$
\mathrm{LR}=\frac{P\left(y_{1} \mid x_{3}, c, n\right)}{P\left(y_{1} \mid x_{3}, \bar{c}, n\right)} \cdot \frac{P\left(r, y_{2} \mid \mathrm{x}_{1}, x_{2}, c, n, g_{2}\right)}{P\left(r, y_{2} \mid x_{1}, x_{2}, \bar{c}, n, g_{2}\right)}
$$

showing that we can treat the fiber evidence (first term) and blood evidence (second term) entirely separately, forming an appropriate likelihood ratio for each and then multiplying. This is because the different kinds of evidence are independent, conditional on either $C=c$ or $C=\bar{c}$.

The above simplification has used just the structure of Fig. 1. However, it is possible to simplify the denominator further, using additional structure, not displayed in the graph, that holds when $C=\bar{c}$, i.e., the suspect was not one of the offenders. In this case it is reasonable further to assume

1. $Y_{1} \Perp\left(X_{3}, N\right) \mid(C=\bar{c})$ : if $\bar{c}$ is the case then the observations on the suspect's jumper, and the number of offenders $N$, have no bearing on the observations on the fibers from the grille.

2. $\left(R, Y_{2}\right) \perp\left(X_{2}, N, G_{2}\right) \mid\left(X_{1}, C=\bar{c}\right):$ if $\bar{c}$ is the case, and knowing the suspect's own blood type, then the properties of the stain of the jumper are not influenced by the guard's evidence, nor the blood type of the guard, nor the number of offenders. So the denominator of (5) simplifies as $P\left(y_{1} \mid \bar{c}\right) P\left(r, y_{2} \mid x_{1} \bar{c}\right)$ and the LR becomes:

$$
\frac{P\left(y_{1} \mid x_{3}, c, n\right)}{P\left(y_{1} \mid \bar{c}\right)} \cdot \frac{P\left(r, y_{2} \mid x_{1}, x_{2}, c, n, g_{2}\right)}{P\left(r, y_{2} \mid x_{1}, \bar{c}\right)}
$$

\section{Evaluation of the LR}

Our assumptions have led us to the idea that the two aspects of the evidence are independent, so we evaluate the fibers and blood evidence separately and combine their contributions to the LR by multiplying them together.

\section{The Fibers Evidence}

First we address the numerator $P\left(y_{1} \mid x_{3}, c, n\right)$. In words this denotes the probability that the tuft of fibers would have the observed properties, given:

$c$ : The suspect was one of the offenders,

$n$ : There were $n$ offenders ( $n=4$ in our example),

$x_{3}$ : The observations on the suspect's jumper.

To proceed with this, it is useful to invoke the unobserved variable $A$ by means of a device which is known as the 'law of total probability' but it is also called the 'law of the extension of the conversation' (see, for example, Lindley (11)). We extend the conversation to include the variable $A$, which has two values: $a$, the suspect was the man who left the tuft; and $\bar{a}$, one of the other men left the tuft. Then the numerator can be written:

$$
\begin{aligned}
P\left(y_{1} \mid x_{3}, c, n\right)= & P\left(y_{1} \mid x_{3}, a, c, n\right) P\left(a \mid x_{3}, c, n\right) \\
& +P\left(y_{1} \mid x_{3}, \bar{a}, c, n\right) P\left(\bar{a} \mid x_{3}, c, n\right)
\end{aligned}
$$

First it can be determined from Fig. 1 that $A \perp \perp X_{3} \mid(C, N)$ and $Y_{1} \perp(C, N) \mid\left(X_{3}, A\right)$. We thus obtain, for the numerator,

$$
P\left(y_{1} \mid x_{3}, a\right) P(a \mid c, n)+P\left(y_{1} \mid x_{3}, \bar{a}\right) P(\bar{a} \mid c, n)
$$

Moreover, although it does not follow from manipulating Fig. 1, it is reasonable to assume in addition that $Y_{1} \perp X_{3} \mid(A=\bar{a})$ : if $\bar{a}$ is the case then $X_{3}$ is irrelevant to the determination of $Y_{1}$. So the numerator simplifies to:

$$
P\left(y_{1} \mid x_{3}, a\right) P(a \mid c, n)+P\left(y_{1} \mid \bar{a}\right) P(\bar{a} \mid c, n)
$$

Note that we have no information, other than $C$ and $N$, to determine the value of $A$. To reflect this state of knowledge we observe that, on the available evidence, the suspect is no more, and no less, likely than any of the other men to have left the tuft of fibers, and so:

$$
\begin{aligned}
& P(a \mid c, n)=1 / n \\
& P(\bar{a} \mid c, n)=(n-1) / n
\end{aligned}
$$

If we now assume that the measurement process which leads to $y_{1}$ and $x_{3}$ is essentially error free, and recall that we have observed the same values for $y_{1}$ and $x_{3}$, then $P\left(y_{1} \mid x_{3}, a\right)$ is approximately one. $P\left(y_{1} \mid \bar{a}\right)$ is the chance that a garment from an unknown person would give measurements $y_{1}$ : Assume that a data survey, such as that described by Laing and Hartshorne (12) enables us to estimate a frequency $f_{1}$ for the occurrence of fibers of the appropriate type among garment fibers. Then the numerator becomes:

$$
\frac{1}{n}+f_{1} \frac{(n-1)}{n}
$$

The denominator, $P\left(y_{1} \mid \bar{c}\right)$ is simply the term $f_{1}$ that has just been defined, so the contribution to the overall LR from the fibers evidence is: $1 / n f_{1}+(1-1 / n)$. In general, $f_{1}$ will be a small number (our numerical example will take the value 0.01 ) so, provided $n$ is not large, the LR for the fibers evidence is approximately

$$
1 / n f_{1}
$$

\section{The Blood Evidence}

The treatment of this evidence leads to a result which is essentially the same as that previously derived by Evett and Buckleton (13) though the detail of the argument used here more closely follows the use of the directed acyclic graph. The numerator of the component due to the blood evidence can also be expanded using the law of the extension of the conversation, this time to include the variable $B$ :

$$
\begin{aligned}
& P\left(r, y_{2} \mid x_{1}, x_{2}, c, n, g_{2}\right) \\
& \quad=P\left(r, y_{2} \mid x_{1}, x_{2}, b, c, n, g_{2}\right) P\left(b \mid x_{1}, x_{2}, c, n, g_{2}\right) \\
& \quad+P\left(r, y_{2} \mid x_{1}, x_{2}, \bar{b}, c, n, g_{2}\right) P\left(\bar{b} \mid x_{1}, x_{2}, c, n, g_{2}\right)
\end{aligned}
$$

This can be simplified by making the following observations from Fig. 1:

2. $\left(R, Y_{2}\right) \perp(C, N) \mid\left(X_{1}, X_{2}, B, G_{2}\right)$. 
Also, though not represented in the graph, it is reasonable to suppose $\left(R, Y_{2}\right) \perp\left(X_{2}, G_{2}\right) \mid\left(X_{1}, B=\bar{b}\right)$.

So:

$$
\begin{aligned}
P\left(r, y_{2} \mid x_{1}, x_{2}, c, n, g_{2}\right)= & P\left(r, y_{2} \mid x_{1}, x_{2}, b, g_{2}\right) P(b \mid c, n) \\
& +P\left(r, y_{2} \mid x_{1}, \bar{b}\right) P(\bar{b} \mid c, n)
\end{aligned}
$$

Following the same line of reasoning as for the fibers evidence:

$$
\begin{aligned}
& P(b \mid c, n)=1 / n \\
& P(\bar{b} \mid c, n)=(n-1) / n .
\end{aligned}
$$

So from the second term of (6) the LR from the blood evidence is

$$
\frac{P\left(r, y_{2} \mid x_{1}, x_{2}, b, g_{2}\right)}{n P\left(r, y_{2} \mid x_{1}, \bar{c}\right)}+\frac{(n-1) P\left(r, y_{2} \mid x_{1}, \bar{b}\right)}{n P\left(r, y_{2} \mid x_{1}, \bar{c}\right)}
$$

Now, $P\left(r, y_{2} \mid x_{1}, \bar{c}\right)=P\left(r, y_{2} \mid x_{1}, b, \bar{c}\right) P(b \mid \bar{c})+P\left(r, y_{2} \mid x_{1}, \bar{b}\right.$, $\bar{c}) P(\bar{b} \mid \bar{c})$, but $P(b \mid \bar{c})=1-P(\bar{b} \mid \bar{c})=0$. So, $P\left(r, y_{2} \mid x_{1}, \bar{c}\right)=P(r$, $\left.y_{2} \mid x_{1}, \bar{b}, \bar{c}\right)$, but from Fig. $1,\left(R, Y_{2}\right) \Perp(C) \mid B$ and this means $P(r$, $\left.y_{2} \mid x_{1}, \bar{c}\right)=P\left(r, y_{2} \mid x_{1}, \vec{b}\right)$ so the blood LR becomes

$$
\frac{P\left(r, y_{2} \mid x_{1}, x_{2}, b, g_{2}\right)}{n P\left(r, y_{2} \mid x_{1}, \bar{b}\right)}+\frac{(n-1)}{n}
$$

The second of the two ratios is less than one and, to a good approximation can be ignored. The first of these two ratios is similar to that considered by Evett and Buckleton (13), apart from the factor $1 / n$. We do not discuss it in detail but write the blood LR approximately as:

$$
\frac{T_{\text {spray }}}{n P_{\text {spray }} f_{2}}
$$

where

$T_{\text {spray }}$ is the probability that a spray of blood would have transferred from the guard to the suspect if he had punched him as described;

$P_{\text {spray }}$ is the probability that a person unconnected to this particular incident would have a spray of blood on his clothing;

$f_{2}$ is the frequency of the observed type of the spray among non-self blood stains on people's clothing.

\section{Numerical Values}

The scientific evidence is thus encapsulated in the LR which is, approximately, the products of (7) and (8):

$$
\frac{T_{\text {spray }}}{n^{2} P_{\text {spray }} f_{1} f_{2}}
$$

Purely for illustration, we take the following values which we consider to be realistic:

$P_{\text {spray: }}:$ From the data collected by Briggs (14) we assign a value of 0.014 ,

$T_{\text {spray }}$ : As explained by Evett and Buckleton (13), this would be a matter for expert judgement, we take 0.5 for illustration, $n$ : We accept the guard's evidence and take a value of 4 , $f_{1}, f_{2}$ : we take both to be 0.01 .

Then the LR is approximately 20,000. We emphasize that we do not consider the precise value of this number to be important-what is important is for the scientist to appreciate the ways in which the various aspects of the evidence interact with each other.

\section{The Prior Evidence}

Finally, having considered the LR term of the right-hand side of (1), we briefly consider the second term, the odds on $C=c$ based on the non-scientific evidence $\left(G_{1}, G_{2}, W\right)$. First, from Fig. $1, C \perp \perp G_{2} \mid\left(G_{1}, W\right)$, so that $P\left(c \mid g_{1}, g_{2}, w\right)=P\left(c \mid g_{1}, w\right)$ and similarly for $\bar{c}$ : The evidence that the guard was punched is not in itself relevant. Second, we have $W \perp G_{1} \mid C$, which, on applying Bayes' theorem, yields:

$$
\frac{P\left(c \mid g_{1}, w\right)}{P\left(\bar{c} \mid g_{1}, w\right)}=\frac{P\left(c \mid g_{1}\right)}{P\left(\bar{c} \mid g_{1}\right)} \cdot \frac{P(w \mid c)}{P(w \mid \bar{c})}
$$

Because we are taking $g_{1}$ as reliable evidence of $N=n$, it seems reasonable to take $P\left(c \mid g_{1}\right)=\alpha n$, where $\alpha$ is a suitable constant of proportionality. Note that this probability (like all others considered) is implicitly conditional on all the remaining evidence in the case, which will affect $\alpha$. If such evidence were essentially absent, we might take $P\left(c \mid g_{1}\right)=n / M$, where $M$ is the size of the population of potential offenders, so that $\alpha=1 / M$. In any event, $\alpha$ is likely to be very small, so that $P\left(c \mid g_{1}\right)$ will be very close to 1 , and the first term in (6) is essentially $\alpha n$. The second term is just the likelihood ratio based on the arresting officer's evidence $W$. Both this, and the constant $\alpha$, must be set by considering non-forensic aspects of the case. Even so, our analysis of the structure of the problem shows just such inputs are required, and how they should combine.

\section{Discussion-Further Development}

We are conscious that many readers of the journal may find the preceding analysis overly complicated and, possibly, difficult to follow. It would be unrealistic to expect the routine use of such methods if they were to be done manually as here; however, we strongly believe, in agreement with Aitken and Gammerman (1), that computer methods are a realistic prospect with existing technology.

One option would be to create a program which did all of the analysis automatically. Indeed, one of us has already participated in the development of such a program for application in a different field which has this capability and we have used it to check the manual analysis. However, we recognize that forensic scientists would not be comfortable with a 'black box' whose inner workings are invisible and not understood. We believe that the solution is an interactive program to assist the scientist step by step through the logical process. The elements of such a program might be as follows.

Graphical Interface-The first requirement is for a graphical interface which enables the scientist to construct the directed acyclic graph which encapsulates the dependencies for each individual case. Such interfaces have already been constructed for other PES and they are well understood. 
Formulation of Starting Expression for the $L R$-The program should assist the user to construct a starting expression for LR in its fullest complexity as at equation 1 , for example.

Advise on Conditional Independencies--The user should be able to propose simplifying independence assumptions which the program will confirm or reject by manipulating the original graph. The user should be able to track the analysis graphically. The program should then update the LR taking account of the new simplification.

Advise on Relevance of Probabilities - The dialogue should in part be based on establishing which of the probabilities the expert feels competent to assign and directing the simplification of the LR in a way which endeavors to make use of assignable probabilities and to avoid those which cannot be assigned.

\section{Acknowledgments}

We wish to thank Dr. CGG Aitken and Professor DV Lindley. They both, independently of each other, made suggestions on a very early draft written by one of us which resulted in our collaboration on this paper.

\section{References}

1. Aitken CGG, Gammerman AJ. Probabilistic reasoning in evidential assessment. J Forensic Sci Soc 1989;29:303-16.

2. Aitken CGG, Stoney DA, editors. The use of statistics in forensic science. Chichester: Ellis Horwood, 1991.

3. Aitken CGG. Statistics and the evaluation of evidence for forensic scientists. Chichester: Wiley, 1995.
4. Robertson B, Vignaux GA. Interpreting evidence: Evaluating forensic science in the courtroom. Chichester: Wiley, 1995.

5. Evett IW. The theory of interpreting scientific transfer evidence. In: Maehly A, Williams RL,-editors. Forensic science progress 4. New York: Springer-Verlag, 1990;141-79.

6. Spiegelhalter DJ, Dawid AP, Lauritzen SL, Cowell RG. Bayesian analysis in Expert Systems. Stat Sci 1993;8:219-83.

7. Wigmore $\mathrm{JH}$. The science of judicial proof: As given by logic, psychology, and general experience and illustrated in judicial trials. 3rd edition. Boston: Little, Brown, 1937.

8. Anderson TJ, Twining WL. Analysis of evidence. London: Weidenfeld and Nicolson, 1991.

9. Buckleton JS, Walsh KAJ. Knowledge based systems. In: Aitken CGG, Stoney DA, editors. The use of statistics in forensic science. Chichester: Ellis Horwood, 1991:186-206.

10. Lauritzen SL, Dawid AP, Larsen BN, Leimer HG. Independence properties of directed Markov fields. Networks 1990;20:491-505.

11. Lindley DV. Probability. In: Aitken CGG, Stoney DA, editors. The use of statistics in forensic science. Chichester: Ellis Horwood, $1991 ; 27-50$.

12. Laing DK, Hartshome AW, Cook R, Robinson G. A fibre data collection for forensic scientists - collection and examination methods. J Forensic Sci 1987;32:364-69.

13. Evett IW, Buckleton J. Some aspects of the Bayesian approach to evidence evaluation. J Forensic Sci Soc 1989;29:317-24.

14. Briggs TJ. The probative value of bloodstains on clothing. Med Sci Law 1978;18:79-83.

Additional information and reprint requests:

Ian W. Evett, D.Sc.

Forensic Science Service

Priory House

Gooch St North

Birmingham B5 6QQ

UK 